\title{
NORMAL CONGRUENCES OF CURVES IN RIEMANN SPACE*
}

BY HARRY LEVY

The purpose of this note is to determine necessary and sufficient conditions that a congruence of curves in a Riemann space of $n$ dimensions be normal to a family of $k$ dimensional hypersurfaces. We proceed first to find certain necessary conditions.

If the hypersurfaces, which we denote for brevity by $V_{k}$, are defined by the equations

$$
f_{i}\left(x^{1} x^{2} \cdots x^{n}\right)=c_{i}, \quad(i=1,2, \cdots, n-k),
$$

where the $c$ 's are constants, any one of the $n-k$ vectors, $\partial f_{i} / \partial x^{n},(i=1,2, \cdots, n-k)$ will be orthogonal to the family. Hence, if a congruence of curves is normal to a family $V_{k}$, there are $n-k$ linearly independent congruences normal to the same family. Define these congruences by the $n-k$ systems of equations

$$
\frac{d x^{1}}{\left.\lambda_{h}\right|^{1}}=\frac{d x^{2}}{\left.\lambda_{h}\right|^{2}}=\cdots=\frac{d x^{n}}{\left.\lambda_{h}\right|^{n}}, \quad(h=1,2,3, \cdots, n-k) .
$$

There will be no loss of generality in assuming that the congruences are mutually orthogonal; if we denote the fundamental tensor of the space by $g_{i j}$, that is, if the linear element is given by the positive definite form

$$
d s^{2}=g_{i j} d x^{i} d x^{j}, \dagger
$$

then we may write

$$
\left.\left.g_{r s} \lambda_{h}\right|^{r} \lambda_{j}\right|^{s}=\delta_{h j}
$$

* Presented to the Society, April 28, 1923.

$\dagger$ An index repeated, once a subscript and once a superscript, is summed from 1 to $n$. 
for $h, j=1,2, \cdots, n-k$, where $\delta_{h j}$ is Kronecker's delta,

$$
\delta_{h j}= \begin{cases}0, & h \neq j, \\ 1, & h=j .\end{cases}
$$

Take any other $k$ congruences, defined by (2) for $h=n-k+1, \cdots, n$, which are mutually orthogonal and orthogonal to the first group. Then (4) will hold for all values of $h$ and $j$ from 1 to $n$.

The coefficients of rotation, $\gamma_{h i j}$, of this orthogonal ennuple will be defined by

$$
\gamma_{h i j}=\left.\left.\lambda_{h \mid r, s} \lambda_{i}\right|^{r} \lambda_{j}\right|^{s} \text {, }
$$

where $\lambda_{h \mid r}$ for $r=1,2, \cdots, n$ are the covariant components of the vector $\lambda_{h \mid}$, that is $\lambda_{h \mid r}=\left.g_{r s} \lambda_{h}\right|^{s}$, and $\lambda_{h \mid r, s}$ is the covariant derivative of $\lambda_{h \mid r}$,

$$
\lambda_{h \mid r, s}=\frac{\partial \lambda_{h \mid r}}{\partial x^{s}}-\lambda_{h \mid t} \Gamma_{r s \mid}{ }^{t}
$$

The functions $\left.\boldsymbol{T}_{r s}\right|^{t}$ are the Christoffel symbols of the second kind.

Ricci has shown $*$ that we may solve (6) for $\lambda_{h \mid r, s}$, and that

$$
\lambda_{h \mid r, s}=\sum_{i, j=1}^{n} \gamma_{h i j} \lambda_{i \mid r} \lambda_{j \mid s} .
$$

If congruences $\lambda_{1 \mid}, \lambda_{2}, \cdots, \lambda_{n-k \mid}$ have a family of $k$-dimensional hypersurfaces as orthogonal trajectories, every direction orthogonal to these $n-k$ congruences must belong to the hypersurface. Hence the equationst

$$
\left.X_{j}(f) \equiv \lambda_{j}\right|^{r} \frac{\partial f}{\partial x^{r}}=0, \quad(j=n-k+1, \cdots, n),
$$

must have $n-k$ independent solutions. A necessary and

* See Méthodes de calcul différentiel absolu, Ricci and Levi-Civita, Mathematische Annalen, vol. 54 (1901), p. 148. As will be seen later, the result of this paper coincides with that of Ricci for the case $k=n-1$.

† Regarding the notation here used, see Goursat, Les Equations Differentielles du Premier Ordre. 
sufficient condition that (9) be a complete system admitting $n-k$ independent solutions is that $\left(X_{i} X_{j}-X_{j} X_{i}\right) f$ be a linear function of the expressions $X_{m}(f)$. Now

$$
X_{i} X_{j}(f)=\left.\lambda_{i}\right|^{r} \frac{\partial}{\partial x^{r}}\left(\left.\lambda_{j}\right|^{s} \frac{\partial f}{\partial x^{s}}\right)=\left.\left.f_{r s} \lambda_{i}\right|^{r} \lambda_{j}\right|^{s}+\left.\left.f_{s} \lambda_{j}\right|_{, r} ^{s} \lambda_{i}\right|^{r}
$$

where

$$
\left.\lambda_{j}\right|_{, r} ^{s}=g^{s t} \lambda_{j \mid t, r},
$$

where $f_{r}$ is $\partial f / \partial x^{r}$, and where $f_{r s}$ is the covariant derivative of $f_{r}$. Making use of (8) to eliminate $\left.\lambda_{j}\right|^{s}, r$, we find, by (4) and (9),

$$
X_{i} X_{j}(f)=\left.\left.f_{r s} \lambda_{i}\right|^{r} \lambda_{j}\right|^{s}+\sum_{l=n-k+1}^{n} \gamma_{j l i} X_{l}+\left.\sum_{h=1}^{n-k} \gamma_{j h i} f_{s} \gamma_{h}\right|^{s} .
$$

Since $f_{r s}$ is symmetric, we have

$$
\left(X_{i} X_{j}-X_{j} X_{i}\right) f=\sum_{l=n-k+1}^{n}\left(\gamma_{j l i}-\gamma_{i l j}\right) X_{l}+\sum_{n=1}^{n-k}\left(\gamma_{j h i}-\gamma_{i h j}\right) f_{s} \lambda_{l} \mid s .
$$

Hence we may write

$$
\left.\sum_{n=1}^{n-k}\left(\gamma_{j h i}-\gamma_{i h j}\right) f_{s} \lambda_{h}\right|^{s}=0 .
$$

All the coefficients of $\left.f_{s} \lambda_{h}\right|^{s}$ must vanish, otherwise we would have another relation between the derivatives of $f$. Consequently, necessary conditions are given by the equations

$$
\begin{gathered}
\gamma_{j h i}-\gamma_{i h j}=0, \\
(h=1,2, \cdots, n-k ; i, j=n-k+1, \cdots, n) .
\end{gathered}
$$

These conditions are also sufficient, hence we have the following theorem.

THEorem. The necessary and sufficient condition that congruences $\lambda_{1}, \lambda_{2}, \cdots, \lambda_{n-k \mid}$ have a family of $k$-dimensional hypersurfaces as orthogonal trajectories is that equations (10) be satisfied. When $k=n-1$ this coincides with Ricci's results.*

* In a paper presented to the International Mathematical Congress in August, 1924, Ricci, unaware of my work, also obtained this general result using essentially the preceding method. 
Suppose every set of $n-k$ congruences of the orthogonal ennuple is normal to a family $V_{k}$. Then equations (10) are satisfied when $h, i$ and $j$ take on any distinct values. But Ricci has shown that $\gamma_{h i j}=-\gamma_{i h j}$; combining, we find $\gamma_{h i j}=0$ for $h, i, j$ distinct, and this is a sufficient condition that all the congruences be normal. Consequently, if every set of $n-k$ congruences $(k>1)$ of an orthogonal ennuple has a family of $k$ dimensional hypersurfaces as orthogonal trajectories, then all of the congruences are normal.

Princeton University

\section{LIMITS FOR ACTUAL DOUBLE POINTS OF SPACE CURVES*}

BY T. R. HOLLCROFT

1. Introduction. Noethert has proved that space curves of maximum genus on non-singular surfaces always exist. In the first part of this paper, such curves will be assumed to exist also when any number of actual double points are added, subject only to the fact that the genus can not be negative for a proper curve and to certain other limitations. The purpose of this paper is to ascertain and define these limitations when all of the actual double points of the space curve are cusps or when all are nodes, and to discuss the existence of space curves with any number of actual double points up to and including the maximum.

From Noether's formula for the maximum genus $\pi_{\mu}$ of a curve of order $n$ on a non-singular surface of order $\mu$, is obtained the theorem: The minimum number of apparent double points $h_{\mu}$ for a space curve of order $n$ cut out by

* Presented to the Society, February 24, 1923.

$\dagger$ M. Noether, Zur Grundlegung der Theorie der Algebraischen Raumkurven, AbHandlungen der Preussischen Akademie DeR Wissenschaften, 1882, Section 6. 